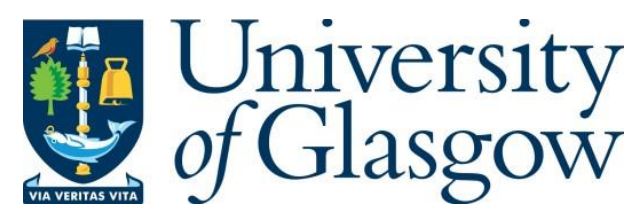

Peveler, W. J., Bear, J. C., Southern, P. and Parkin, I. P. (2014) Organic-inorganic hybrid materials: nanoparticle containing organogels with myriad applications. Chemical Communications, 50(92), pp. 14418-14420.

There may be differences between this version and the published version. You are advised to consult the publisher's version if you wish to cite from it.

http://eprints.gla.ac.uk/150509/

Deposited on: 20 November 2017

Enlighten - Research publications by members of the University of Glasgow http://eprints.gla.ac.uk 


\title{
Organic-Inorganic Hybrid Materials: Nanoparticle Containing Organogels with Myriad Applications ${ }^{\dagger}$
}

\author{
William J. Peveler, ${ }^{a, b}$, Joseph C. Bear ${ }^{* b}$, Paul Southern, ${ }^{c}$ and Ivan P. Parkin, ${ }^{b}$
}

Received Xth $X X X X X X X X X X 20 X X$, Accepted $X$ th $X X X X X X X X X 20 X X$

First published on the web Xth $X X X X X X X X X X 200 X$

DOI: $10.1039 / b 000000 x$

The synthesis of hybrid inorganic/organic materials from a single-component organogelator is reported. Varied functional inorganic materials were included and the resultant physico-chemical properties of the gels are presented. These materials are quick, versatile, can be cast into virtually any form, and the nanoparticles are easily reclaimed.

Low molecular weight organogelators (LMWOs) are fascinating materials with a wide range of applications, being lightweight, optically clear, self-healing and and easily mouldable $^{1}$. The LMWO material is dissolved in a suitable solvent at elevated temperature, and on cooling self assembles into a fibrous network, creating the gel. Organogels have been used as a method for encapsulating nanomaterials with good spatial organisation and stability, leading to the development of optoelectronic devices, catalytic frameworks and new magnetic materials ${ }^{2-6}$. However, few previous works utilise preformed nanomaterials in gels, and of these, many use complex or multi-component gelators, increasing system complexity when compared to a LMWO.

In this work we report the synthesis of a range of functional hybrid "smart" materials. These are fabricated by combining a recently-described, mannitol-based LMWO, 1, with a single step, one hour synthesis and the highly desirable physical properties described above, in addition to low toxicity ${ }^{7,8}$, with straight-from-the-reactor nanoparticles in their solvent, 1-octadecene (ODE). This quickly created gels with fluorescent, magnetic and other properties. The nanoparticles can be stabilised by the gel, and may even confer mechanical stability $^{9,10}$. They are easily inserted and reclaimed, with the gelator 1 being fully recyclable. Finally, the gel medium can also act as a surfactant for a new in-situ nanoparticle synthesis. The

$\dagger$ Electronic Supplementary Information (ESI) available: [Experimental procedures and characterisation data for all new materials, additional TEM and EDS, recycling procedures and hot cast structures with functional properties. ]. See DOI: 10.1039/b000000x/

${ }^{a}$ Department of Security and Crime Science, University College London,, London, WC1H 9EZ

${ }^{b}$ Department of Chemistry, University College London, London, WC1H OAJ; E-mail: joseph.bear.11@ucl.ac.uk

${ }^{c}$ UCL Healthcare Biomagnetics Laboratories, Royal Institution of Great Britain, 21 Albemarle Street, London, WIS 4BS, UK

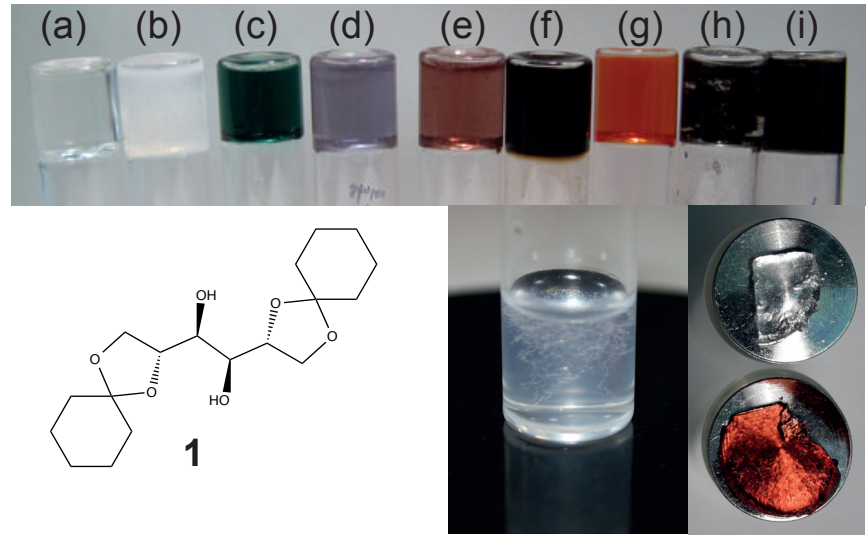

Fig. 1 Top: Images of different nanoparticle gels at $3 \mathrm{wt} \%$ loading unless noted, (a) Plain ODE gel, (b) $10 \mathrm{wt} \%$ ODE gel, (c) CoO NP gel, (d) TOAB AuNP gel, (e) DDT AuNP gel, (f) $\mathrm{Fe}_{3} \mathrm{O}_{4}$ NP gel, (g) $\mathrm{CdSe} / \mathrm{ZnS}$ QD gel, (h) virgin MWCNT gel and (i) functionalised MWCNT gel. Bottom left: Structure of mannitol gelator 1. Bottom centre: Growth of fibrous network during gelation of $10 \%$ ODE gel. Bottom right: Samples of plain 4 wt\% ODE gel before and after Au sputtering.

gels created are easily recast in any shape and show excellent longevity.

The full syntheses are given in the Supporting Information $\dagger$, but briefly, to create a typical gel, between 0.5 and $10 \mathrm{wt} \%$ (weight percent) of gelator 1 was dissolved at $65^{\circ} \mathrm{C}$ in a $1 \mathrm{ml}$ sample of the inorganic material of interest in ODE (the reaction solvent for formation of many of the NPs). On cooling, gelation was tested by inversion. ODE solutions of trioctylphosphine (TOP) capped CdSe/ZnS QDs, tetraoctylammonium bromide (TOAB) stabilised AuNPs, dodecanethiol (DDT) stabilised AuNPs, TOP stabilised Ni NPs, oleic acid (OA) stabilised $\mathrm{Fe}_{3} \mathrm{O}_{4}$ NPs, OA stabilised $\mathrm{CoO}$ NPs, and multi-wall carbon nanotubes (MWCNTs) were all successfully converted into gels (stable to inversion) with $3 \mathrm{wt} \%$ or less of gelator $\mathbf{1}$.

Physicochemical characterisation of all the gels was carried out, including Scanning Electron Microscopy (SEM) and Tunnelling Electron Microscopy (TEM) imaging of the distribu- 




Fig. 2 TEM imagary of selected nanoparticle gels: (a) CoO NPs in gel matrix, (b) DDT stabilised AuNPs in gel matrix, (c) QDs in gel matrix,

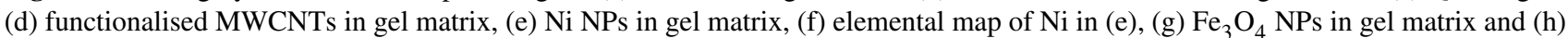
AuNPs as synthesised in a plain $4 \mathrm{wt} \%$ gel.

tions of nanomaterials within the fibrous network. In each case a small portion of the gel was cut with a scalpel and imaged. TEM images (Fig. 2) show a good dispersion of the NPs within the gel, often confined within the fibrous nature of the gel, which showed up well over the holey-carbon film TEM grid (Agar Scientific). Energy filtered transmission electron microscopy was used to illustrate the presence of Ni NPs in the gel as evidenced by the zero loss electron micrograph and $\mathrm{Ni}$ edge map in Fig. 2e\&f. Oxygen edge mapping was also used to show that $\mathrm{NiO}$ was not present, indicating that no oxidation occurs to the NPs on gel formation (ESI $\dagger$ ). A sample of the gel without nanoparticles was imaged under environmental SEM (see ESI $\dagger$ ), and showed the largely smooth macrostructure of the material, with some fibrous microstructure. On drying the material under ultra-high vacuum, the sample was re-imaged via field-emission SEM, and the fibrous structure was better resolved. The melting point of the ODE gel was $65^{\circ} \mathrm{C}$, and the critical gelation concentration (CGC) was $0.357 \mathrm{wt} \%$.

Fluorescent gels were produced by the introduction of TOP capped CdSe/ZnS quantum dots into the ODE solvent (Fig. 1g, 2c), and showed little alteration to their optical properties, other than a slight reduction of fluorescence intensity, likely caused by the gel material scattering some of the incident and emissive light (Fig. 3A). These QD gels were also gas permeable, as evidenced with a sensing experiment for toxic VOC nitrobenzene. A thin film of the QD gel on a glass slide was exposed to $197 \mathrm{ppm}$ of nitrobenzene (natural vapour pressure of a toxic VOC) and fluorescence quenching was observed after $120 \mathrm{~s}$ (Fig. 3B).

Metal oxide NPs capped with oleylamine were amenable to incorporation into the gels. Cobalt oxide nanostructures (wurtzite ${ }^{11}$-golf-tee shapes - Fig. 2a) were used to create a attractive green gel (Fig. 1c) and a magnetic gel was created from superparamagnetic ferrite NPs in ODE (Fig. 1f, 2g). A variety of strengths were created by varying the NP concentration and gelator concentration and its properties were probed by SQUID measurements (Fig. 3C). Although direct comparison between measured samples was difficult, data normalised against the maximum saturation magnetisation indicated that there was negligible impact of gelator concentration on magnetisation of a sample, suggesting that even high gelator concentrations create very stable magnetic gels with little or no detrimental effect on the particle properties.

Pure metal nanoparticles were also compatible with the gel, when coated with a range of surfactants. AuNPs coated with TOAB or DDT were successfully incorporated, with the TOAB stabilised NPs remaining in a transparent gel long after the non-gelled solution had precipitated out (Fig. 1d\&e, 2b). An interesting observation was mild aggregation of TOAB stabilised AuNPs in the gel, as evidenced by a red-shift in the ab- 

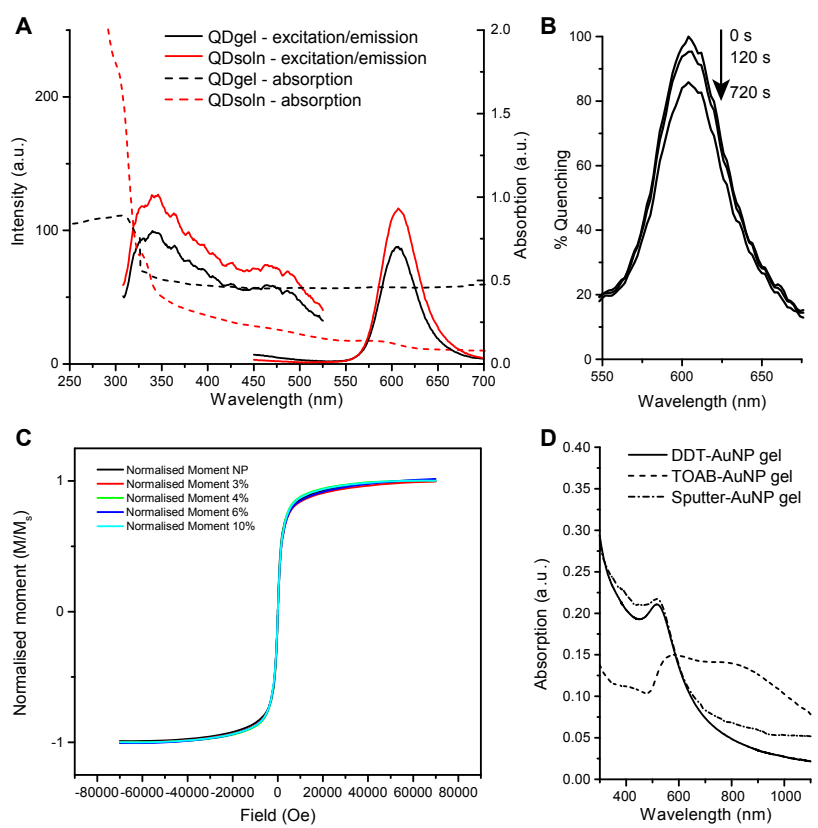

Fig. 3 A: Fluorescence emission (ex:365 nm) and excitation (em:605 nm) and absoprtion spectra (dashed) for gel and ungelled solution. B: Thin film of QD gel exposed to nitrobenzene vapour for varying time. C: SQUID measurments show magnetic moment normalised against maximum moment for various wt $\%$ gels and the raw magnetic NPs. D: Absorption of various AuNP gels created in different ways.

sorption on gelling in comparison to the DDT stabilised material (Fig. 3D) and TEM (ESI $\dagger$ ). This suggests an ionic interaction between the charge stabilised NPs and the gelator, which is not exhibited by the DDT stabilised particles. Pure $\mathrm{Ni}$ nanoparticles were also successfully incorporated into the gel (Fig. 2e\&f and ESI $\dagger$ ), and were tested as a supported catalyst for production of $\mathrm{H}_{2}$ from aqueous $\mathrm{NH}_{3} \mathrm{BH}_{3}$ solution ( 0.4 M). ${ }^{12,13}$ Catalytic activity was observed at the phase boundary between hydrophobic gel and the solution, and $32 \mathrm{ml}$ of $\mathrm{H}_{2}$ was produced over 24 hours.

It was also discovered that pure metal nanoparticles could be generated in-situ within the gel matrix. Samples of gel were exposed to gold sputter (experimental details in ESI $\dagger$ ) and remained clear after treatment with a few spots of gold. A strong red colour then developed after 24 hours (Fig. 1 - bottom right). TEM confirmed the presence of remarkably uniform AuNPs trapped in the gel matrix, in high concentration, without disrupting the structure of the gel (Fig. 2h), and the narrow SPR band indicated good monodispersity (Fig. 3D). The process was repeated with a silver sputter target and similar results were achieved (details in ESI $\dagger$ ). It is hoped in the future this material might be able to act as a Raman signal enhancing substrate via a SERS mechanism.
MWCNTs were incorporated into the gel via simple mixing, however as the gel cooled some clumping occurred (Fig. 1h). To reduce this phenomenon, the MWCNTs were coated with a pyrene conjugate via a literature procedure and thus became fully dispersible in $\mathrm{ODE}^{14}$. These could then be set into a long lasting and mechanical stable gel (Fig. 1i, 2d). Indeed shapes cast from these gels maintained their form for far longer than those cast from other nanomaterial gels, suggesting mechanical stabilisation of the matrix (see $\mathrm{ESI} \dagger$ ).

This work demonstrates the ease that nanoparticles in a non-polar solvent of synthesis can be gelled straight from the pot, without the requirement for phase transfers or ligand exchanges. The enforced separation of the NPs prevents loss of suspension, and the particles can be easily reclaimed via ethanol precipitation and centrifugation for further solution phase applications (see ESI $\dagger$ ).

The gels demonstrated here have low CGC, and useful melting temperature rendering it easy to gel, melt, and re-gel. The LMWO itself is easy to synthesise on a large scale, with minimal workup and a long shelf life, giving it advantages over other peptide and dendrimer LMWOs, and requires only a single component, offering advantages over micellular systems. There is also possibility to combine several elements within the gel to create multi-modal systems, combining many physical characteristics into one inexpensive and robust material, with applications in optical sensing, nanoparticle facilitated catalysis and nanoparticle synthesis and stabilisation.

\section{Acknowledgement}

The authors thank Miss Emma Newton for assistance with the photography (Fig. 1: Bottom centre) and Dr Michael B. Ward at the Leeds EPSRC Nanoscience and Nanotechnology Facility for access to elemental mapping TEM. This work was supported in part by EPSRC Grant no: EP/G037264/1 as part of UCL's Security Science Doctoral Training Centre.

\section{References}

1 M. Cametti and Z. Džolić, Chem. Commun., 2014.

2 P. D. Wadhavane, R. E. Galian, M. A. Izquierdo, J. Aguilera-Sigalat, F. Galindo, L. Schmidt, M. I. Burguete, J. Pérez-Prieto and S. V. Luis, J. Am. Chem. Soc., 2012, 134, 20554-20563.

3 K. Trickett, H. Brice, O. Myakonkaya, J. Eastoe, S. E. Rogers, R. K. Heenan and I. Grillo, Soft Matter, 2010, 6, 1291-1296.

4 P.-E. Le Renard, O. Jordan, A. Faes, A. Petri-Fink, H. Hofmann, D. Rüfenacht, F. Bosman, F. Buchegger and E. Doelker, Biomaterials, 2010, 31, 691-705.

5 E. Taboada, L. N. Feldborg, A. Pérez del Pino, A. Roig, D. B. Amabilino and J. Puigmartí-Luis, Soft Matter, 2011, 7, 2755.

6 S. K. Samanta, A. Pal, S. Bhattacharya and C. N. R. Rao, J. Mater. Chem., 2010, 20, 6881-6890.

7 A. Vidyasagar, K. Handore and K. M. Sureshan, Angew. Chem. Int. Ed. Engl., 2011, 50, 8021-8024. 
8 A. Prathap and K. M. Sureshan, Chem. Commun., 2012, 48, 5250-5252.

9 D. Zhang, J. Yang, S. Bao, Q. Wu and Q. Wang, Sci Rep, 2013, 3, 1399.

10 C. Liao, Q. Wu, T. Su, D. Zhang, Q. Wu and Q. Wang, ACS Appl. Mater. Interfaces, 2014, 6, 1356-1360.

11 M. R. Buck, A. J. Biacchi and R. E. Schaak, Chem. Mater, 2014, 26, 1492-1499.

12 C. R. Crick, J. C. Bear, P. Southern and I. P. Parkin, J. Mater. Chem. A, 2013, 1, 4336.

13 P.-Z. Li, A. Aijaz and Q. Xu, Angew. Chem. Int. Ed. Engl., 2012, 51, 6753-6756.

14 R. J. Colchester, C. A. Mosse, D. S. Bhachu, J. C. Bear, C. J. Carmalt, I. P. Parkin, B. E. Treeby, I. Papakonstantinou and A. E. Desjardins, Appl. Phys. Lett., 2014, 104, 173502. 